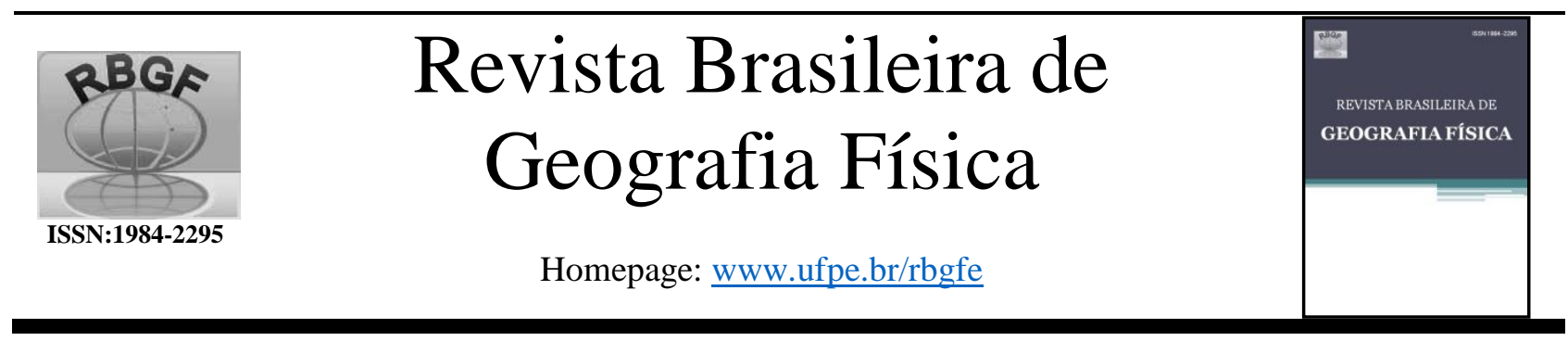

\title{
Comparação entre dados meteorológicos provenientes de sensoriamento remoto (modelados e de satélites) e de estações de superfície
}

\author{
Nuria Aparecida Miatto Rampazo- Universidade Estadual de Campinas, autor correspondente e-mail: \\ nuriarampazo@gmail.com, Michelle Cristina Araujo Picoli-Instituto Nacional de Pesquisas Espaciais, Carla Kazue \\ Nakao Cavaliero- Instituto Nacional de Pesquisas Espaciais
}

\begin{abstract}
R E S U M O
Dados meteorológicos obtidos por sensoriamento remoto são uma alternativa para suprir a escassez de informações provindas de estações meteorológicas convencionais e automáticas. O objetivo deste trabalho consistiu em comparar dados meteorológicos derivados de satélites (CERES, GLDAS, CM SAF) e modelos (PERSIANN, ECMWF) com dados observados em 31 estações meteorológicas (INMET) do estado de São Paulo, no período de janeiro de 2015 a junho de 2016. Para verificar a eficiência dos dados oriundos de modelos e satélites em relação aos dados de estações meteorológicas, foram calculados o teste de normalidade de Shapiro-Wilk, coeficiente de correlação de Spearman, Erro Médio (mean error, ME), Erro Médio Absoluto (mean absolute error, MAE) e Índice de Concordância de Willmott modificado (d1). De modo geral, os resultados obtidos através dos testes estatísticos indicaram que as estimativas fornecidas pelos produtos ou dados de modelos analisados são alternativas adequadas para aplicação em estudos climáticos, com exceção do dado de umidade relativa, que não apresentou boa exatidão em relação aos dados de estação meteorológica.
\end{abstract}

Palavras-chave: CERES; CM SAF; GLDAS; PERSIANN; ECMWF.

\section{Comparison of meteorological data obtained by remote sensing (from models and satellites) and weather stations data}

\begin{abstract}
A B S T R A C T
Meteorological data obtained by remote sensing are an alternative to supply the gap of information proceeded from conventional and automatic weather stations. The aim of this study was to compare meteorological data derived from satellites (CERES, GLDAS, CM SAF) and models (PERSIANN, ECMWF) with observed data in 31 weather stations (INMET) of the state of São Paulo, during the period January 2015 to June 2016. The efficiency of the data from models and satellites in relation to weather stations data was evaluated by the following tests: Shapiro-Wilk normality test, Spearman's correlation, mean error (ME), mean absolute error (MAE) and modified Willmott index of agreement (d1). In general, the results obtained through the statistical tests indicated that the estimates provided by the analyzed products or data from models are suitable alternatives for application in climatic studies, with the exception of the relative humidity data, which did not show good accuracy in relation to weather station data.
\end{abstract}

Keywords: CERES; CM SAF; GLDAS; PERSIANN; ECMWF. 


\section{Introdução}

A importância de se analisar variáveis meteorológicas decorre do fato que tais elementos afetam o ambiente como um todo, trazendo implicações para a sociedade em todas as atividades humanas, com destaque para a agricultura (GuhaSapir et al., 2015; Xavier et al., 2016). Situado na região sudeste do Brasil, o estado de São Paulo teve 8.479.029 hectares ocupados por cultivos agrícolas perenes ou temporários (IBGE, 2015), o que equivale a $34 \%$ de sua área territorial (IBGE, 2010), e é justamente desta extensão do território ocupada pela agricultura que decorre a importância do monitoramento e análise de variáveis meteorológicas. Informações precisas e com boa cobertura espacial e temporal são essenciais para a produção agrícola, pois permitem o monitoramento do desenvolvimento de culturas, gestão hídrica adequada, dentre outros.

Apesar de São Paulo ser um dos estados brasileiros com maior cobertura de dados meteorológicos, esta cobertura é ainda escassa e espacialmente descontínua, além de ser sujeita a apresentar falhas temporais nos dados disponibilizados (Baba et al., 2014; Depiné et al., 2014; Xavier et al., 2016). Precipitação pluvial, radiação solar, temperatura, umidade do ar e velocidade do vento são elementos climáticos que exercem direta e grande influência sobre $o$ crescimento e a produtividade de culturas agrícolas, atuando sobre as taxas de evapotranspiração (Allen et al., 1998).

Neste contexto caracterizado pela baixa densidade de estações meteorológicas, alto custo de equipamentos e na logística de disponibilização de dados, produtos provindos de satélites meteorológicos e estimativas de modelos regionais e globais surgem como uma ferramenta de grande utilidade para suprir a falta de informação convencional e subsidiar a análise da ocorrência espacial e temporal de elementos climáticos (Camparotto et al., 2013; Andrade et al., 2016; Xavier et al., 2016). Assim, permitem um planejamento mais adequado das atividades agrícolas, bem como estimar sua produtividade. Além disso, estações meteorológicas possuem um caráter pontual que não é capaz de captar a variabilidade espacial de variáveis meteorológicas (Camparotto et al., 2013), o que prejudica diretamente o planejamento da agricultura.

Dessa forma, a hipótese do trabalho consiste no fato de que dados climáticos provenientes de sensoriamento remoto podem substituir dados climáticos convencionais e, por conseguinte, confirmar se os dados de sensoriamento remoto são robustos para sua utilização em estimativas, Rampazo, N. A. M., Picoli, M. C. A., Cavaliero, C. K. N. monitoramento climático e agrícola, dentre outros. Para isso, é necessário verificar a eficiência ou a acurácia de dados provenientes de sensores orbitais e de modelos antes de sua utilização. Acurácia ou exatidão refere-se ao "grau de concordância entre um valor medido e um valor verdadeiro dum mensurando" (INMETRO, 2012), aqui considerando o valor medido como o dado proveniente de sensoriamento remoto e o valor verdadeiro como o dado observado em estação meteorológica. Deste modo, o objetivo deste trabalho consistiu em comparar dados meteorológicos de estações meteorológicas com dados derivados de satélites e modelos para o estado de São Paulo. Devido à disponibilidade dos produtos modelados ou de satélite utilizados, a análise foi subdividida em duas escalas temporais: diária e decendial - na primeira, foram comparados dados diários de radiação solar, temperatura do ar, umidade relativa, velocidade do vento e precipitação de superfície (estações meteorológicas do Instituto Nacional de Meteorologia (INMET)) com dados derivados de satélites (CERES, GLDAS, CM SAF) e modelos globais (PERSIANN, ECMWF); na segunda, foram comparados dados decendiais de radiação solar, temperatura do ar e precipitação das estações meteorológicas do INMET com dados de um modelo regional. A seleção das variáveis meteorológicas foi feita com base na disponibilidade das mesmas, tanto nas estações do INMET como em dados derivados de modelos e de satélites disponíveis gratuitamente na Internet.

\section{Material e métodos}

Como parâmetro para a comparação, dados pontuais provindos de 31 estações meteorológicas automáticas de superfície no estado de São Paulo (Figura 1) foram obtidos junto ao INMET, para as variáveis radiação solar, temperatura do ar, umidade relativa, velocidade do vento e precipitação. Os dados do INMET são disponibilizados em escala horária e dados horários faltantes foram preenchidos com a média dos dados anterior e posterior à falha (Johann et al., 2011), com exceção dos dados de precipitação (neste caso, um dado horário faltante eliminou o dia todo, já que a precipitação é um dado acumulado). Após o preenchimento de falhas horárias, os dados do INMET foram agrupados para a escala diária (acompanhado de preenchimento de falhas diárias) e também para a escala decendial (três decêndios por mês, iniciados nos dias 01, 11 e 21), para que pudessem se comparados com os dados do modelo ECMWF. O período analisado, de janeiro de 2015 a junho de 2016, totalizou 547 dias e 54 decêndios. Os pontos correspondentes às estações meteorológicas do INMET foram utilizados para a 
extração dos dados derivados de satélites e de modelos globais e regionais em ambiente GIS (Geographical Information System). A grade espacial dos dados utilizados é apresentada na Figura 1.

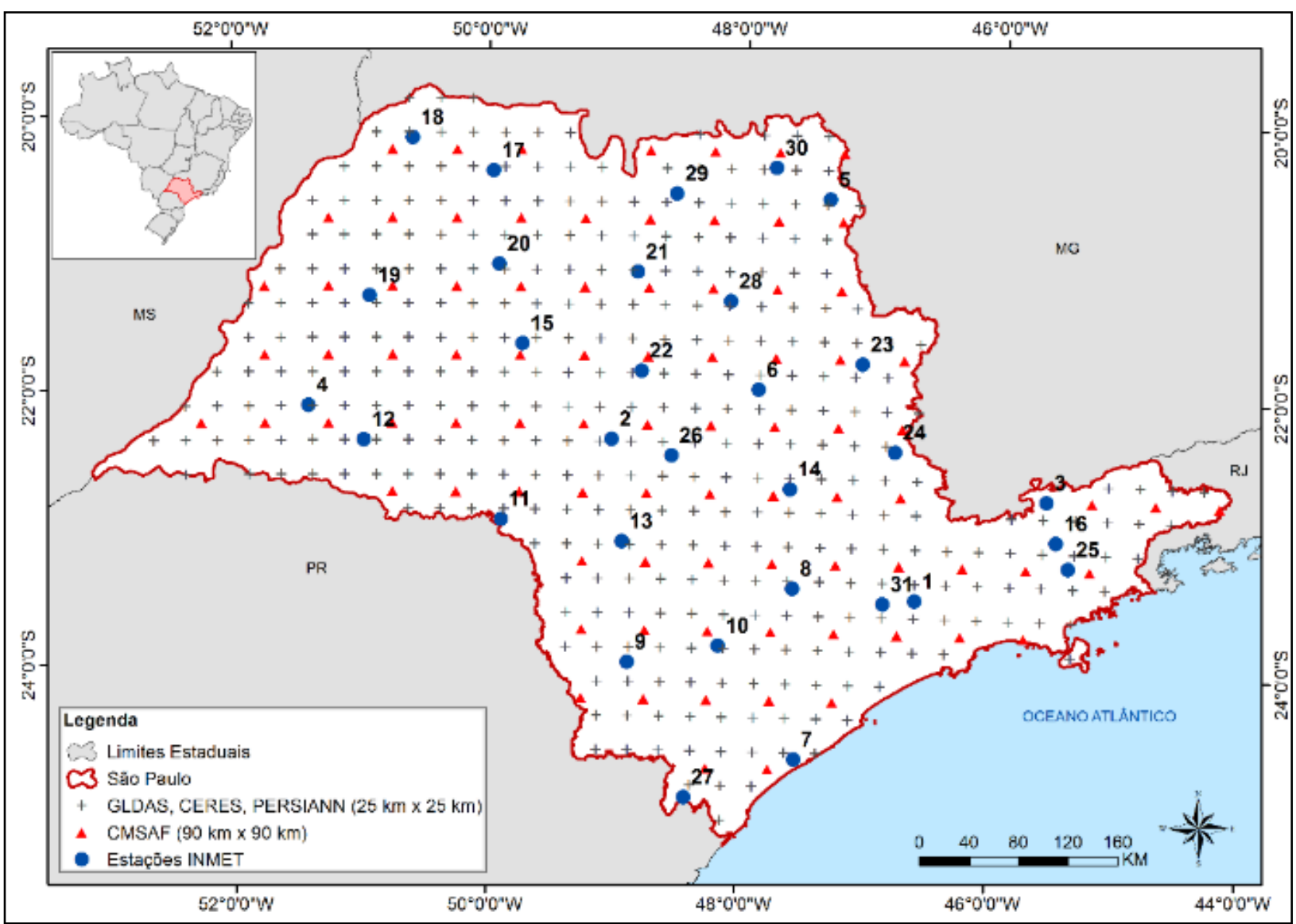

Figura 1. Área de estudo e grade espacial dos dados climáticos comparados.

Dados diários de radiação solar foram obtidos a partir do experimento climatológico Clouds and the Earth's Radiant Energy System (CERES; Wielicki et al., 1996; disponível em: <https://neo.sci.gsfc.nasa.gov/view.php?datasetId= CERES_NETFLUX_D>. Acesso em 03 mai. 2017), que possui instrumentos a bordo dos satélites Aqua e Terra, do projeto FLASHFlux da National Aeronautics and Space Administration (NASA). Os instrumentos do CERES são do tipo varredura termal, medem a radiação solar refletida e a radiação de ondas longas emitida pela Terra (Wielicki et al., 1996), e possuem três canais: um total $(0.3-100 \mu \mathrm{m})$, um de ondas curtas $(0.3-5 \mu \mathrm{m})$ e um de ondas longas $(8-12 \mu \mathrm{m})$ (Szewczyk et al., 2005). O experimento CERES disponibiliza dados de saldo de radiação $\left(\mathrm{W} / \mathrm{m}^{2}\right.$ ), com resolução espacial de $0,25^{\circ}$ (cerca de $25 \times 25 \mathrm{~km}^{2}$ ) e resolução temporal diária, que foram então extraídos e convertidos para radiação solar global $\left(\mathrm{kJ} / \mathrm{m}^{2}\right)$ com base em Davies (1967).

Dados de temperatura do ar e velocidade do vento foram obtidos através de um produto do Rampazo, N. A. M., Picoli, M. C. A., Cavaliero, C. K. N. sistema de assimilação de dados da NASA Global Land Data Assimilation System (GLDAS; Rodell et al., 2004; disponível em: <https://disc.sci.gsfc.nasa.gov/>. Acesso em 20 jan. 2017.), com resolução espacial de $0,25^{\circ}$ (cerca de 25 x $25 \mathrm{~km}^{2}$ ) e resolução temporal de 3 horas (produto GLDAS_NOAH025_3H_2.0). O sistema gera dados modelados da superfície terrestre a partir de técnicas de modelagem e assimilação de produtos de satélite e dados observados em superfície (Rodell et al., 2004). Após a extração, os dados foram convertidos para a escala diária. Os dados de velocidade do vento são disponibilizados em $\mathrm{m} / \mathrm{s}$ e os dados de temperatura do ar em $\mathrm{K}$, sendo o último convertido para ${ }^{\circ} \mathrm{C}$.

Dados diários de umidade relativa foram obtidos do centro Satellite Application Facility on Climate Monitoring (CM SAF; Schulz et al., 2009; disponível em: <https://wui.cmsaf.eu/>. Acesso em 20 jan. 2017), através do produto HLW do sensor ATOVS (Advanced TIROS Operational Vertical Sounder), com resolução espacial de $90 \times 90$ km² e 
resolução temporal diária. O produto HLW utilizado apresenta resolução temporal diária correspondente à média diária (Schulz et al., 2009). O CM SAF pertence à European Organisation for the Exploitation of Meteorological Satellites (EUMETSAT) e tem como objetivo a produção de dados para o monitoramento climático derivados de através da exploração com algoritmos de medições feitas por instrumentos a bordo de satélites (Schulz et al., 2009; EUMETSAT, 2017).

Os dados diários de precipitação foram provenientes do modelo Precipitation Estimation from Remotely Sensed Information using Artificial Neural Networks (Persiann; Hsu et al., 1997; disponível em: <http://chrsdata.eng.uci.edu/>. Acesso em 20 jan. 2017), desenvolvido pela Universidade do Arizona, com resolução espacial de $0,25^{\circ}$ (cerca de $25 \times 25 \mathrm{~km}^{2}$ ) e resolução temporal diária. $\mathrm{O}$ modelo foi inicialmente calibrado com dados infravermelhos coletados pelo satélite Tabela 1. Fonte dos Dados Meteorológicos comparados
Geostationary Meteorological Satellite (GMS) e dados meteorológicos coletados em superfície (Hsu et al., 1997).

Dados decendiais de radiação solar, temperatura do ar e precipitação foram obtidos pelo modelo produzido pelo European Center for Medium-Range Weather Forecast (ECMWF; JRC, 2017; disponível em: $<$ http://spirits.jrc.ec.europa.eu/>. Acesso em 20 jan. 2017), com resolução espacial de $0,25^{\circ}$ (cerca de 25 x $25 \mathrm{~km}^{2}$ ). $\mathrm{O}$ modelo agrega e redefine estimativas de chuva, radiação solar, evapotranspiração e temperatura do ar para algumas regiões do globo, como África, Ásia, Europa, Pacífico e Américas do Norte e do Sul (JRC, 2017).

As fontes e as resoluções espaciais dos dados meteorológicos derivados de satélites e de modelos podem ser conferidas na Tabela 1 .

\begin{tabular}{lll}
\hline Variável Meteorológica & \multicolumn{1}{c}{ Escala Diária } & \multicolumn{1}{c}{ Escala Decendial } \\
\hline Radiação Solar & CERES $\left(25 \times 25 \mathrm{~km}^{2}\right)$ & $\begin{array}{l}\text { ECMWF }(25 \times 25 \\
\left.\mathrm{km}^{2}\right)\end{array}$ \\
Temperatura do Ar & GLDAS $\left(25 \times 25 \mathrm{~km}^{2}\right)$ & $\begin{array}{l}\text { ECMWF }(25 \times 25 \\
\left.\mathrm{km}^{2}\right)\end{array}$ \\
Umidade Relativa & CM SAF $\left(90 \times 90 \mathrm{~km}^{2}\right)$ & - \\
Velocidade do Vento & GLDAS $\left(25 \times 25 \mathrm{~km}^{2}\right)$ & - \\
Precipitação & $\begin{array}{l}\text { PERSIANN }(25 \times 25 \\
\left.\mathrm{km}^{2}\right)\end{array}$ & $\begin{array}{l}\text { ECMWF }(25 \times 25 \\
\left.\mathrm{km}^{2}\right)\end{array}$ \\
\hline
\end{tabular}

Os dados inicialmente foram submetidos a uma análise de outliers a fim de verificar a existência de dados discrepantes. Os limites diários considerados para cada variável meteorológica são apresentados na Tabela 2, bem como as referências utilizadas. Importante ressaltar que os valores máximos para os dados decendiais de radiação solar e precipitação consideraram dez vezes os valores dos limites diários, já que estes são dados acumulados.

Tabela 2. Limites considerados para a análise de outliers

\begin{tabular}{ccc}
\hline Variável & Limites & Referência \\
\hline Radiação Solar (RS) & $0<\mathrm{RS}<41.000$ & Adaptado de Tiba et al. (2001) \\
Temperatura do Ar (TA) & $-14<\mathrm{TA}<+50{ }^{\circ} \mathrm{C}$ & Baba et al. (2014), INMET (2009) \\
Umidade Relativa (UR) & $0<\mathrm{UR}<100 \%$ & Baba et al. (2014) e INMET (2009) \\
Velocidade do Vento & $0<\mathrm{VV}<35 \mathrm{~m} / \mathrm{s}$ & Adaptado de INMET (2009) e Liska et al. \\
(VV) & $0<\mathrm{PP}<140 \mathrm{~mm}$ & $(2013)$ \\
Precipitação (PP) & INMET (2009) \\
\hline
\end{tabular}

Para verificar a eficiência dos dados estimados por modelos e oriundos de satélites em relação aos dados observados nas estações meteorológicas (INMET), foram calculados o teste de normalidade de Shapiro-Wilk (Shapiro \& Wilk, 1965) (Eq. 1), coeficiente de correlação de Spearman (Spearman, 1904) (Eq. 2), Erro Médio (mean error,
ME) (Eq. 3), Erro Médio Absoluto (mean absolute error, MAE) (Eq. 4) e Índice de Concordância de Willmott modificado (d1; Willmott et al., 1985) (Eq. 5). Os testes de Shapiro-Wilk e correlação de Spearman foram analisados com um nível de significância de $5 \%(\alpha=0,05)$. Foi utilizado o índice de Willmott modificado (d1) ao invés do original 
(d2; Willmott, 1981) porque, por utilizar a função quadrática, o índice $\mathrm{d} 2$ pode resultar em valores elevados, mesmo quando o modelo estimador não apresenta performance satisfatória (Willmott et al., 1985). O índice d1 varia de 0 a 1 , com valores próximos a 1 indicando maior concordância entre os dados observados (de estações meteorológicas) e estimados (dados de sensoriamento remoto).

$$
W=\frac{b^{2}}{\sum_{i=1}^{n}\left(x_{i}-x\right)^{2}}
$$

Onde: "xi" refere-se aos valores da amostra ordenados e "b" é uma constante.

$$
\rho=1-\frac{6 \sum_{i=1}^{n} d_{i}^{2}}{\left(n^{3}-\mathrm{n}\right)}
$$

Onde: "di" corresponde à diferença entre cada posição das variáveis x e y e " $n$ " corresponde ao número dos pares dos valores.

$$
\begin{gathered}
M E=n^{-1} \sum_{i=1}^{n} s_{i}-m \\
M A E=n^{-1} \sum_{i=1}^{n}\left|s_{i}-m_{i}\right| \\
d_{1}=1-\left[\frac{\sum\left(\left|m_{i}-s_{i}\right|\right)}{\sum\left(\left|m_{i}-o\right|+\left|s_{i}-s\right|\right)}\right]
\end{gathered}
$$

Nas Equações 3 a 5, "s" refere-se aos dados observados nas estações meteorológicas de superfície (INMET) e "m" aos dados estimados por satélites e modelos regionais e globais.

Por fim, os resultados do índice de concordância de Willmott modificado (d1) foram espacializados através do método Empirical Bayesian Kriging (EBK) em ambiente GIS, para possibilitar a visualização da variabilidade espacial da concordância entre os dados derivados de satélites e modelos e de estações meteorológicas de superfície no estado de São Paulo.

\section{Resultados e discussão}

A Tabela 3 mostra a porcentagem de dados faltantes (diários e decendiais) para as variáveis meteorológicas radiação solar (RS), temperatura do ar (TA), umidade relativa (UR), velocidade do vento (VV) e precipitação (PP). O período analisado (de janeiro de 2015 a junho de 2016) totaliza 547 dados diários e 54 dados decendiais. Devido à insuficiência de dados válidos, os pontos a seguir foram retirados da análise de algumas variáveis: pontos 22 (temperatura do ar e velocidade do vento, dados diários) e 31 (radiação solar, dados diários e decendiais), respectivamente correspondentes às estações de Ibitinga e Barueri.

A normalidade dos dados meteorológicos de estação e dos dados derivados de modelos e satélites foi examinada com o teste de Shapiro-Wilk (com nível de significância de 5\%). Como esperado, os dados diários não apresentaram distribuição normal (p-valores inferiores a 0,05), com exceção dos pontos 3 (Campos do Jordão) e 18 (Jales) para velocidade do vento; e do ponto 25 (São Luiz do Paraitinga) para temperatura do ar. Importante ressaltar que o resultado de Campos do Jordão está relacionado à quantidade de dados faltantes de velocidade do vento $(58,3 \%$, como pode ser visto na Tabela 3). Os p-valores para o teste de normalidade dos dados decendiais foram superiores ao nível de significância de $5 \%(\alpha=0,05)$, o que, no entanto, não leva à conclusão de que os dados não possuem distribuição normal, já que o teste de Shapiro-Wilk é sensível ao tamanho da amostra e este fato pode ter interferido nos resultados: para o período analisado, são 547 dados diários contra 54 dados decendiais.

As Tabelas 6 e 7 apresentam os resultados médios para o coeficiente de correlação de Spearman $(\rho)$, erro médio (ME), erro médio absoluto (MAE) e índice de concordância de Willmott modificado (d1), calculados entre os dados das fontes comparadas (estações meteorológicas e dados derivados de modelos e satélites).

A correlação média verificada pelo coeficiente de correlação de Spearman $(\alpha=0,05)$ entre as fontes comparadas de dados diários (Tabela 6) foi igual a 0,57 para os dados de radiação solar, 0,82 para temperatura do ar, 0,42 para umidade relativa, 0,41 para velocidade do vento e 0,63 para precipitação. Em relação aos dados decendiais do modelo ECMWF (Tabela 7), os resultados do coeficiente de correlação de Spearman $(\alpha=0,05)$ indicaram correlação média superior a 0,90 para radiação solar e temperatura do ar e correlação média de 0,80 para o dado de precipitação.

Com base nos erros médios (ME) constatouse que os dados diários derivados de modelos e satélites superestimaram a radiação solar em 100,0\% dos pontos e a velocidade do vento em $80,0 \%$ dos pontos, enquanto subestimaram a umidade relativa em $100,0 \%$ dos pontos. A temperatura foi subestimada em $50,0 \%$ e superestimada em $50,0 \%$ dos pontos e a precipitação foi subestimada em $58,1 \%$ dos pontos. A média dos erros médios para os dados diários (Tabela 6) foi igual a 5.295,45 ( \pm $1.793,57) \mathrm{kJ} / \mathrm{m}^{2} /$ dia para radiação solar, 0,05 ( \pm $2,24){ }^{\circ} \mathrm{C}$ para temperatura do ar, -40,97 $( \pm 5,58) \%$ para umidade relativa, $0,48( \pm 0,57) \mathrm{m} / \mathrm{s}$ para velocidade do vento e entre $-0,12( \pm 0,74) \mathrm{mm}$ para precipitação (Tabela 6). 
Tabela 3. Pontos analisados e quantidade (\%) de dados faltantes

\begin{tabular}{|c|c|c|c|c|c|c|c|c|c|c|}
\hline \multirow[t]{2}{*}{ Ponto } & \multirow[t]{2}{*}{ Estação } & \multirow[t]{2}{*}{ Município } & \multicolumn{5}{|c|}{ \% Dados Diários Faltantes (Total: 547) } & \multicolumn{3}{|c|}{$\begin{array}{l}\text { \% Dados Decendiais } \\
\text { Faltantes (Total: } 547 \text { ) }\end{array}$} \\
\hline & & & RS & TA & UR & VV & $\mathbf{P P}$ & RS & TA & PP \\
\hline 1 & A701 & São Paulo & $1,8 \%$ & $0,0 \%$ & & $0,0 \%$ & $0,7 \%$ & $0,0 \%$ & $0,0 \%$ & $3,7 \%$ \\
\hline 2 & A705 & auru & & $0 \%$ & & & & $0,0 \%$ &, $0 \%$ & $14,8 \%$ \\
\hline 3 & A706 & C. do Jordão & & $0 \%$ & $34,7 \%$ & & $0,5 \%$ & $0,0 \%$ & $0,0 \%$ & $5,6 \%$ \\
\hline 4 & A707 & $\begin{array}{l}\text { Pres. } \\
\text { Prudente }\end{array}$ & $8,4 \%$ & $11,2 \%$ & $11,3 \%$ & $11,2 \%$ & $13,2 \%$ & $13,0 \%$ & $3,7 \%$ & $24,1 \%$ \\
\hline 5 & A708 & Franca & $1,8 \%$ & $0,0 \%$ & 0,4 & $0,0 \%$ & $0,4 \%$ & $0,0 \%$ & $0,0 \%$ & $1,9 \%$ \\
\hline 6 & & S & & & & & & $9,3 \%$ & $7,4 \%$ & $11,1 \%$ \\
\hline 7 & $A$ & & $\%$ & $9,3 \%$ & & & $9,3 \%$ & $9,3 \%$ & $7,4 \%$ & $11,1 \%$ \\
\hline 8 & 3 & & & & & & $0,2 \%$ & $1,9 \%$ & $0,0 \%$ & $1,9 \%$ \\
\hline 9 & & Itape & & $10,1 \%$ & & $9,3 \%$ & $11,0 \%$ & $11,1 \%$ & $7,4 \%$ & $14,8 \%$ \\
\hline 10 & & $\begin{array}{l}\text { S. Miguel } \\
\text { Arc. }\end{array}$ & $1,8 \%$ & $0,0 \%$ & $0,4 \%$ & $0,0 \%$ & $0,2 \%$ & $0,0 \%$ & $0,0 \%$ & $1,9 \%$ \\
\hline 11 & & & $9,5 \%$ & $7,7 \%$ & & $7,7 \%$ & $16,5 \%$ & $9,3 \%$ & $5,6 \%$ & $27,8 \%$ \\
\hline 12 & & & 4,0 & & & & & $5,6 \%$ & & $50,0 \%$ \\
\hline 13 & & & & & & & & $0,0 \%$ & $\%$ & $3,7 \%$ \\
\hline 14 & & & & & & & 10 & $11,1 \%$ & $\%$ & $18,5 \%$ \\
\hline 15 & & & & & & & & $11,1 \%$ & 7,4 & $14,8 \%$ \\
\hline 16 & & & & 11, & 12 , & 11 , & 11 , & 20,4 & $9,3 \%$ & $13,0 \%$ \\
\hline 17 & & & & & & & & 0,0 & $0,0 \%$ & $3,7 \%$ \\
\hline 18 & 733 & & 7 & $13,9 \%$ & 13,7 & $14,1 \%$ & $17,9 \%$ & $0,0 \%$ & $13,0 \%$ & $25,9 \%$ \\
\hline 19 & A734 & Valparaî & $5,3 \%$ & $3,5 \%$ & $3,8 \%$ & $3,1 \%$ & $4,0 \%$ & $3,7 \%$ & $1,9 \%$ & $9,3 \%$ \\
\hline 20 & A735 & $\begin{array}{l}\text { José } \\
\text { Bonifácio }\end{array}$ & $1,8 \%$ & $12,8 \%$ & $13,2 \%$ & $12,8 \%$ & $14,8 \%$ & $0,0 \%$ & $9,3 \%$ & $20,4 \%$ \\
\hline 21 & A736 & Ariran & $10,6 \%$ & $10,1 \%$ & $10,4 \%$ & $10,1 \%$ & $19,0 \%$ & $11,1 \%$ & $7,4 \%$ & $22,2 \%$ \\
\hline 22 & A737 & Ibitin & $1,8 \%$ & $100,0 \%$ & $0,4 \%$ & $100,0 \%$ & $0,4 \%$ & $0,0 \%$ & $0,0 \%$ & $3,7 \%$ \\
\hline 23 & A738 & Casa Branc & $6,4 \%$ & $5,5 \%$ & $5,9 \%$ & $5,5 \%$ & $13,9 \%$ & $3,7 \%$ & $3,7 \%$ & $20,4 \%$ \\
\hline 24 & A739 & Itapira & $3,1 \%$ & $3,5 \%$ & $3,8 \%$ & $3,5 \%$ & $4,6 \%$ & $5,6 \%$ & $1,9 \%$ & $9,3 \%$ \\
\hline 25 & A740 & $\begin{array}{l}\text { S. L. } \\
\text { Paraitinga }\end{array}$ & $6,6 \%$ & $4,8 \%$ & $5,1 \%$ & $4,8 \%$ & $4,8 \%$ & $3,7 \%$ & $1,9 \%$ & $5,6 \%$ \\
\hline 26 & A741 & Barra Bonita & $11,2 \%$ & $9,7 \%$ & $10,1 \%$ & $9,7 \%$ & $12,8 \%$ & $13,0 \%$ & $7,4 \%$ & $31,5 \%$ \\
\hline 27 & A746 & $\begin{array}{l}\text { Barra do } \\
\text { Turvo }\end{array}$ & $1,8 \%$ & $0,0 \%$ & $0,4 \%$ & $0,0 \%$ & $13,5 \%$ & $0,0 \%$ & $0,0 \%$ & $48,1 \%$ \\
\hline 28 & & & & & & & & $0,0 \%$ & $0,0 \%$ & $46,3 \%$ \\
\hline 29 & & & & $0,0 \%$ & & $0,0 \%$ & $0,2 \%$ & $0,0 \%$ & $0,0 \%$ & $1,9 \%$ \\
\hline 30 & & & $1,8 \%$ & $7,5 \%$ & $7,9 \%$ & $7,5 \%$ & $7,7 \%$ & $0,0 \%$ & $7,4 \%$ & $9,3 \%$ \\
\hline 31 & A755 & Barueri & $96,0 \%$ & $6,0 \%$ & $6,4 \%$ & $6,0 \%$ & $6,6 \%$ & $96,3 \%$ & $5,6 \%$ & $11,1 \%$ \\
\hline
\end{tabular}

Em relação aos dados decendiais, os valores dos erros médios (ME) indicaram que os dados de radiação solar e temperatura foram superestimados pelo modelo ECMWF em $86,7 \%$ e em $74,2 \%$ dos pontos, respectivamente, enquanto a precipitação foi superestimada em 50,0\% dos pontos e subestimada em 50\% dos pontos. Para os dados decendiais, a média dos erros médios foi igual a 12.433,16 ( \pm $16.623,03) \mathrm{kJ} / \mathrm{m}^{2} /$ dia para radiação solar, 0,32 $( \pm$ $0,62){ }^{\circ} \mathrm{C}$ para temperatura do ar e $2,49( \pm 8,52) \mathrm{mm}$ para precipitação (Tabela 7). Importante ressaltar Rampazo, N. A. M., Picoli, M. C. A., Cavaliero, C. K. N. que os dados decendiais de radiação solar e precipitação são dados acumulados para o período de dez dias.

Foram encontrados erros médios absolutos (MAE) de 5.982,76 $( \pm 1.274,45) \mathrm{kJ} / \mathrm{m}^{2} / \mathrm{dia}$ para radiação solar, 2,15 $( \pm 1,22){ }^{\circ} \mathrm{C}$ para temperatura do ar, 41,01 $( \pm 5,54) \%$ para umidade relativa, $0,79( \pm$ $0,33) \mathrm{m} / \mathrm{s}$ para velocidade do vento e $4,25( \pm 0,95)$ mm para precipitação para os dados diários (Tabela 6). Para os dados decendiais, os valores médios de MAE foram $19.648,49( \pm 13.356,16) \mathrm{kJ} / \mathrm{m}^{2} /$ dia para 
radiação solar, $0,77( \pm 0,51){ }^{\circ} \mathrm{C}$ para temperatura do ar e 23,52 $( \pm 3,83) \mathrm{mm}$ para precipitação (Tabela 7). Cabe observar que os valores de MAE são superiores aos valores encontrados pelo $\mathrm{ME}$, isto porque no cálculo do erro médio (Eq. 3) diferenças negativas

Tabela 4. Limites verificados nos dados meteorológicos diários

\begin{tabular}{|c|c|c|c|c|}
\hline \multirow[b]{2}{*}{ Variável Meteorológica } & \multicolumn{2}{|c|}{ Estações INMET } & \multicolumn{2}{|c|}{ Satélites e Modelos } \\
\hline & Mínimo & Máximo & Mínimo & Máximo \\
\hline $\begin{array}{ll}\text { Radiação } & \text { Solar } \\
\left(\mathrm{kJ} / \mathrm{m}^{2} / \mathrm{dia}\right) & \end{array}$ & 406,50 & $34.069,40$ & $8.482,91$ & $35.345,45$ \\
\hline Temperatura do $\operatorname{Ar}\left({ }^{\circ} \mathrm{C}\right)$ & 4,20 & 31,82 & 4,38 & 32,54 \\
\hline Umidade Relativa (\%) & 18,54 & 100,00 & $-1,47$ & 73,73 \\
\hline $\begin{array}{l}\text { Velocidade do Vento } \\
(\mathrm{m} / \mathrm{s})\end{array}$ & 0,00 & 7,33 & 0,39 & 6,17 \\
\hline Precipitação (mm) & 0,00 & 136,40 & 0,00 & 128,40 \\
\hline
\end{tabular}

Tabela 5. Limites verificados nos dados meteorológicos decendiais

\begin{tabular}{|c|c|c|c|c|}
\hline \multirow[b]{2}{*}{$\begin{array}{l}\text { Variável } \\
\text { Meteorológica }\end{array}$} & \multicolumn{2}{|c|}{ Estações INMET } & \multicolumn{2}{|c|}{ Satélites e Modelos } \\
\hline & Mínimo & Máximo & Mínimo & Máximo \\
\hline $\begin{array}{ll}\text { Radiação } & \text { Solar } \\
\left(\mathrm{kJ} / \mathrm{m}^{2} / \mathrm{dia}\right) & \end{array}$ & $11.214,40$ & $293.094,30$ & $66.715,00$ & $294.610,00$ \\
\hline Temperatura do $\operatorname{Ar}\left({ }^{\circ} \mathrm{C}\right)$ & 8,84 & 29,8 & 11,18 & 29,91 \\
\hline Precipitação (mm) & 0,00 & 389,40 & 0,00 & 329,00 \\
\hline
\end{tabular}

Os erros verificados estão de acordo com os resultados encontrados na literatura. Wielicki et al. (2002) analisaram a acurácia de dados de satélite para o balanço de radiação, incluindo os instrumentos do CERES, para os quais a acurácia verificada foi de $1 \%\left(1 \mathrm{~W} / \mathrm{m}^{2}\right.$ para o período de duas décadas analisado. Dessler (2010) também trabalhou com o produto do instrumento CERES e, adicionando uma tendência de $\pm 0,5 \mathrm{~W} / \mathrm{m}^{2} /$ década, verificou o impacto de uma incerteza estatística nos dados do CERES, a qual totalizou 0,74 e 0,77 $\mathrm{W} / \mathrm{m}^{2} / \mathrm{K}$. Segundo o autor, este erro nos dados pode estar relacionado ao efeito decorrente da entre a nebulosidade e a temperatura do ar.

De Gonçalves et al. (2006) avaliaram três dos mais importantes produtos de sensoriamento remoto de precipitação diária para a América do Sul, dentre eles o modelo PERSIANN. Os autores verificaram que o produto do modelo PERSIANN apresentou tendência de subestimar áreas sem precipitação e superestimar áreas com volumes de precipitação inferiores a $5 \mathrm{~mm}$, além de apresentar dificuldade na localização de áreas com e sem ocorrência de precipitação. No entanto, os autores concluíram que o modelo PERSIANN apresentou melhor comportamento que outros produtos avaliados.

Moazami et al. (2013) avaliaram três produtos de satélites de precipitação diária, dentre Rampazo, N. A. M., Picoli, M. C. A., Cavaliero, C. K. N. são anuladas por diferenças positivas, o que não ocorre no cálculo do erro médio absoluto (Eq. 4), que utiliza a função módulo. eles o PERSIANN, para o Irã e concluíram que o PERSIANN tendeu a subestimar a precipitação, apresentando comportamento erros maiores que os outros dois produtos analisados (TMPA-3B42V7 e TMPA-3B42RT).

Decker et al. (2011) avaliaram diversos produtos de reanálise, dentre os quais os modelos ECMWF e GLDAS. Para os produtos de temperatura do ar, os autores encontraram um erro de 2,71 $( \pm 1,43)$ para o ECMWF e de $2,79( \pm 3,64)$ para o GLDAS. Em relação à velocidade do vento, o produto do GLDAS apresentou um erro de 2,93 ( \pm 3,93 ). O ECMWF apresentou ainda erros de 3,50 ( \pm $2,29)$ para o produto de precipitação e de 3,15 ( \pm 2,38) para o produto de radiação solar.

Haiden et al. (2016) investigaram subestimativas no novo modelo do ECMWF, indicando suas melhorias em relação às versões anteriores. Segundo os autores, o produto de temperatura do ar foi melhorado, com uma diminuição de $3 \%$ e $1 \%$ no erro RMSE, respectivamente para $o$ hemisfério norte extratropical e para os trópicos. Em relação ao produto de precipitação, o erro RMSE não indicou melhorias, enquanto houve uma pequena degradação de $1 \%$ nos dados para os trópicos, verificada a partir da pontuação SEEPS (Stable Equitable Error in Probability Space). 
Para os dados de temperatura do ar do ECMWF para o Paraná (Brasil), Blain et al. (2006) obtiveram $\mathrm{R}^{2}=0,75$, enquanto Johann et al. (2011) obtiveram $\mathrm{R}^{2}=0,79$, com um erro médio variando entre $-1,81$ e $+2,33{ }^{\circ} \mathrm{C}$, concluindo que o modelo analisado informa com bastante precisão a temperatura média do ar.
O uso desses dados sem correção poderia impactar em erros de estimativas grandes, já que, dependendo do dado meteorológico, o erro pode chegar até 100\%. Para amenizar esses erros, é possível fazer uma correção dos mesmos, como observado em trabalhos de Johann et al. (2011).

Tabela 6. Resultados dos testes de correlação ( $\rho$ ), erro médio (ME), erro médio absoluto (MAE) e índice de concordância de Willmott modificado (d1) para os dados diários - Média e desvio padrão das 31 estações analisadas

\begin{tabular}{lcrrr}
\hline Variável Meteorológica & \multicolumn{1}{c}{$\boldsymbol{\rho}^{*}$} & \multicolumn{1}{c}{ ME } & \multicolumn{1}{c}{ MAE } & \multicolumn{1}{c}{ d1 } \\
\hline Radiação Solar $\left(\mathrm{kJ} / \mathrm{m}^{2} / \mathrm{dia}\right)$ & $0,57( \pm 0,14)$ & $5.295,45( \pm 1.793,57)$ & $5.982,76( \pm 1.274,45)$ & $0,46( \pm 0,07)$ \\
Temperatura do Ar $\left({ }^{\circ} \mathrm{C}\right)$ & $0,82( \pm 0,17)$ & $0,05( \pm 2,24)$ & $2,15( \pm 1,22)$ & $0,63( \pm 0,14)$ \\
Umidade Relativa $(\%)$ & $0,42( \pm 0,09)$ & $-40,97( \pm 5,58)$ & $41,01( \pm 5,54)$ & $0,17( \pm 0,05)$ \\
Velocidade do Vento $(\mathrm{m} / \mathrm{s})$ & $0,41( \pm 0,17)$ & $0,48( \pm 0,57)$ & $0,79( \pm 0,33)$ & $0,36( \pm 0,12)$ \\
Precipitação $(\mathrm{mm})$ & $0,63( \pm 0,07)$ & $-0,12( \pm 0,74)$ & $4,25( \pm 0,95)$ & $0,65( \pm 0,08)$ \\
\hline
\end{tabular}

${ }^{*}$ Significante a 5\% $(\alpha=0,05)$. Obs. A unidade indicada entre parênteses refere-se aos valores de ME e MAE; $\rho$ e d1 são adimensionais.

Tabela 7. Resultados dos testes de correlação ( $\rho$ ), erro médio (ME), erro médio absoluto (MAE) e índice de concordância de Willmott modificado (d1) para os dados decendiais - Média e desvio padrão das 31 estações analisadas

\begin{tabular}{lcrrr}
\hline Variável Meteorológica & \multicolumn{1}{c}{$\boldsymbol{\rho}^{*}$} & \multicolumn{1}{c}{ ME } & \multicolumn{1}{c}{ MAE } & \multicolumn{1}{c}{ d1 } \\
\hline Radiação Solar $\left(\mathrm{kJ} / \mathrm{m}^{2} / \mathrm{dia}\right)$ & $0,90( \pm 0,17)$ & $12.433,16( \pm$ & $19.648,49( \pm$ & $0,73( \pm 0,16)$ \\
Temperatura do Ar $\left({ }^{\circ} \mathrm{C}\right)$ & $0,93( \pm 0,19)$ & $0,32( \pm 0,62)$ & $0,77( \pm 0,51)$ & $0,82( \pm 0,12)$ \\
Precipitação $(\mathrm{mm})$ & $0,80( \pm 0,07)$ & $2,49( \pm 8,52)$ & $23,52( \pm 3,83)$ & $0,66( \pm 0,05)$ \\
\hline
\end{tabular}

${ }^{*}$ Significante a $5 \%(\alpha=0,05)$. Obs. A unidade indicada entre parênteses refere-se aos valores de ME e MAE; $\rho$ e d1 são adimensionais.

Entre as variáveis analisadas, o índice de Willmott modificado (d1) indicou, para escala diária, que a precipitação pluvial (Tabela 10) e a temperatura do ar (Tabela 7) são os elementos que apresentaram a maior concordância com os dados de superfície $(\mathrm{d} 1$ médio $=0,65$ e 0,63 , respectivamente), enquanto a radiação solar (Tabela 6) apresentou d 1 médio igual a 0,46 , a velocidade do vento (Tabela 9) d1 médio igual a 0,36 e, por fim, a umidade relativa (tabela 8) apresentou a menor concordância $(\mathrm{d} 1$ médio $=0,17)$. Já para os dados decendiais, obtiveram-se valores médios de d1 iguais a 0,73 para radiação solar (Tabela 11), 0,82 para temperatura do ar (Tabela 12) e 0,66 para precipitação (Tabela 13), indicando boa concordância entre os dados modelados e os dados observados nas estações de superfície.

A Figura 2 mostra a variabilidade espacial dos valores interpolados pelo método EBK do índice de concordância de Willmott modificado (d1) no estado de São Paulo para os dados diários. Através do índice de Willmott modificado verificou-se que os dados de radiação solar, temperatura do ar e precipitação apresentaram a maior concordância entre os dados modelados e de superfície, enquanto os dados de umidade relativa e de velocidade do vento apresentaram os resultados mais baixos. Para a radiação solar, o índice d1 apresentou valores mais elevados na região noroeste $(0,45<\mathrm{d} 1<0,50)$ e mais baixos $(0,40<\mathrm{d} 1<0,45)$ no sudeste do estado de São Paulo (Figura 2a). Os dados de temperatura do ar apresentaram os maiores resultados $(\mathrm{d} 1>0,65)$ na região sul do estado, e os mais baixos $(0,50<\mathrm{d} 1<$ $0,55)$ no extremo oeste do estado (Figura $2 b$ ). Para os valores de umidade relativa (Figura 2c), os maiores resultados $(0,20<\mathrm{d} 1<0,25)$ foram verificados na região noroeste e diminuem em direção ao sul e sudeste $(0,10<\mathrm{d} 1<0,15)$. Já para 
velocidade do vento, o índice d1 apresentou valores mais altos $(0,35<\mathrm{d} 1<0,40)$ em direção ao sul e ao noroeste e valores mais baixos $(0,30<\mathrm{d} 1<0,35) \mathrm{em}$ direção ao nordeste e leste do estado (Figura 2d). Por fim, para precipitação os índices foram maiores $(\mathrm{d} 1$ $>0,70)$ em direção sudoeste e menores $(0,60<\mathrm{d} 1<$ $0,65)$ em direção ao norte e ao extremo leste do estado (Figura 2e).

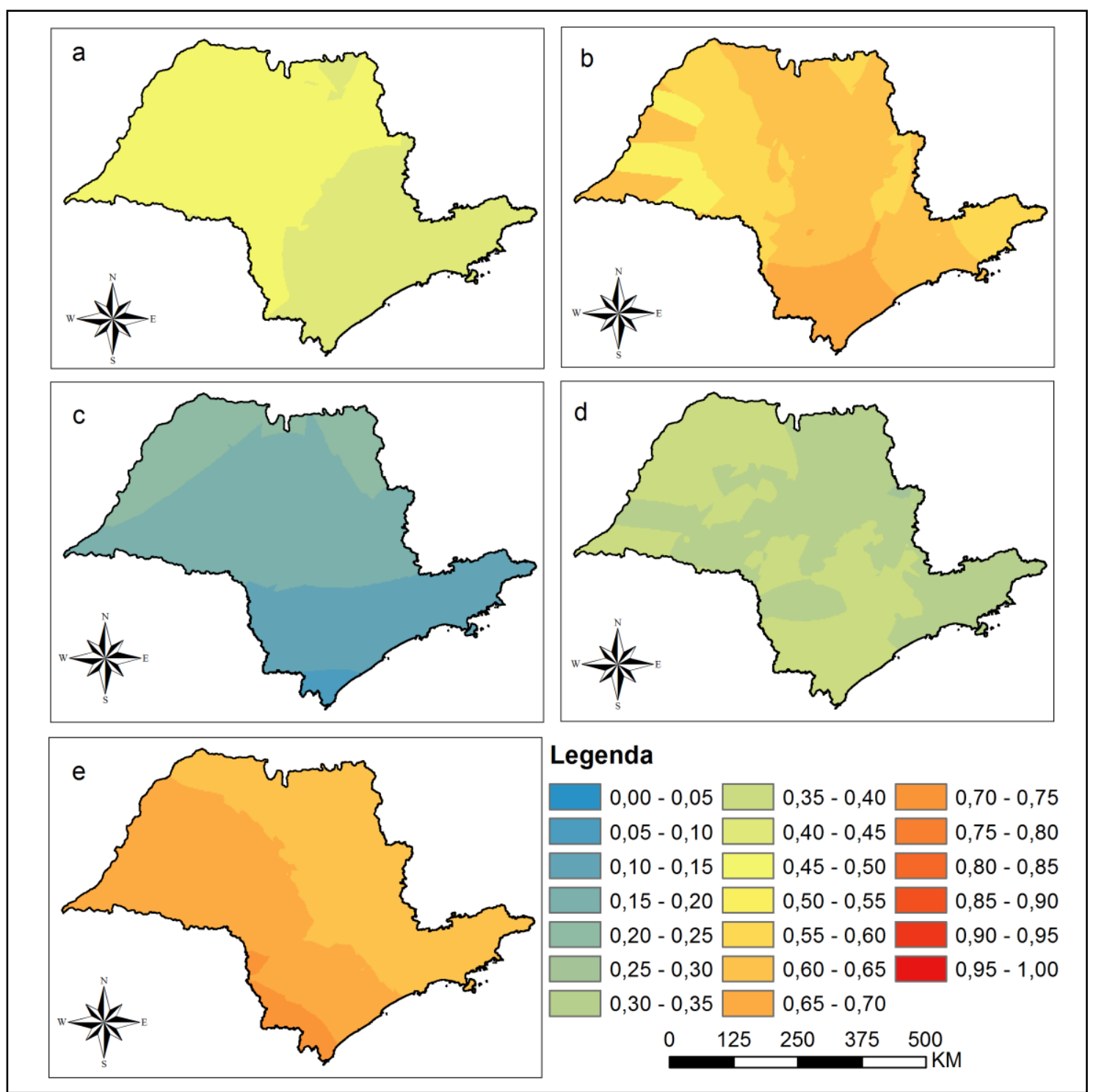

Figura 2. Índice de Willmott modificado para os dados diários de radiação solar (a), temperatura do ar (b), umidade relativa (c), velocidade do vento (d) e precipitação (e).

Comparando-se a Figura 2 com o relevo do estado de São Paulo, Martinelli, 2009, constatou-se que o dado de radiação solar (CERES) apresentou melhor desempenho em direção ao noroeste do estado, onde o relevo é plano e apresenta menores altitudes. O desempenho dos dados de temperatura do ar e velocidade do vento (ambos do sistema GLDAS) apresentou comportamento oposto ao da radiação solar, com maiores índices em direção ao sul do estado, onde o relevo é acidentado e elevado. O comportamento do desempenho do dado de umidade relativa (CM SAF) apresentou relação com as zonas climáticas do estado de São Paulo Rolim et al., 2007. Por sua vez, o modelo PERSIANN (precipitação) não apresentou relação aparente com o clima e nem com o relevo do estado.

A Figura 3 apresenta a variabilidade espacial dos valores interpolados do índice de Willmott modificado (d1) para os dados decendiais do ECMWF, no estado de São Paulo, confirmando a concordância verificada entre os dados modelados e dados de estações meteorológicas de superfície (d1 médio igual a $0,73,0,82$ e 0,66 , respectivamente para radiação solar, temperatura do ar e precipitação). $\mathrm{O}$ índice $\mathrm{d} 1$ apresentou valores superiores em direção ao sul do estado para radiação solar $(\mathrm{d} 1>0,70)$ e 
temperatura do ar $(\mathrm{d} 1>0,85)$, enquanto o índice para precipitação apresentou baixa variabilidade espacial no estado $(0,65<\mathrm{d} 1<0,70)$, observando-se leve diminuição nos valores em direção ao extremo leste $(0,60<\mathrm{d} 1<0,65)$.

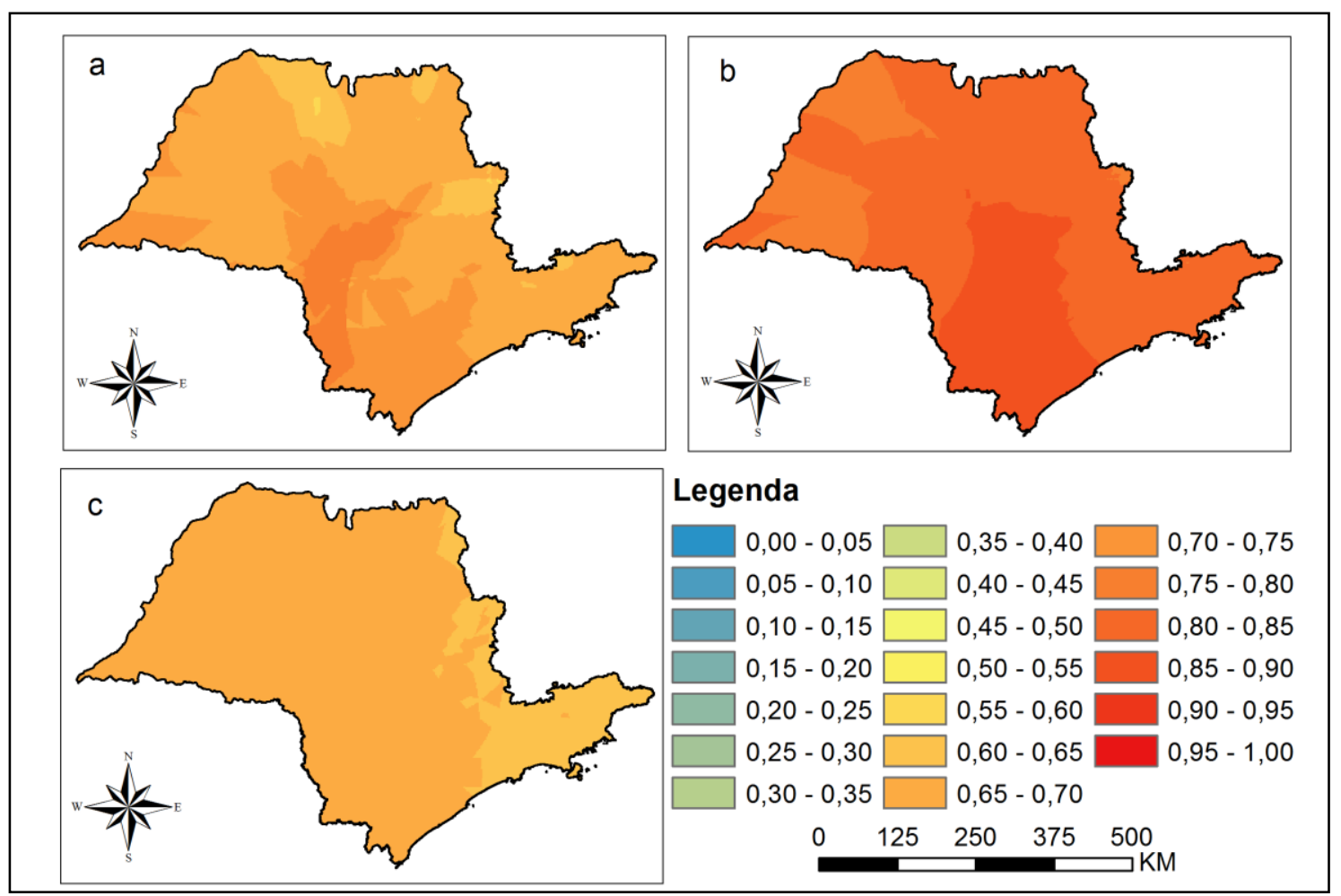

Figura 3. Índice de Willmott modificado para os dados decendiais de radiação solar (a), temperatura do ar (b) e precipitação (c)

Comparando-se a Figura 3 com o relevo do estado de São Paulo, Martinelli, 2009, constatou-se que os dados decendiais de radiação solar e temperatura do ar do modelo ECMWF apresentaram melhor desempenho em direção ao sul do estado, o relevo é acidentado e elevado. Já o desempenho do dado decendial de precipitação do ECMWF apresentou baixa variabilidade espacial no estado.

A Figura 4 exemplifica o comportamento dos modelos de regressão linear simples (RLS) para o ponto 10 (São Miguel Arcanjo) - ponto com menor quantidade de dados faltantes (Tabela 3). A linha contínua é a linha de tendência nos dados apresentados e a linha pontilhada é a reta 1:1. Destaca-se o ajuste entre os dados diários modelados ou de satélites e de estação meteorológica para as variáveis temperatura do ar (Figura $4 \mathrm{~b}$, com $\mathrm{R}^{2}=$ 0,7496 e d1 $=0,65$ ) e precipitação (Figura $4 \mathrm{e}$, com $\mathrm{R}^{2}=0,384$ e d $\left.1=0,66\right)$ e entre os dados decendiais modelados e de estação meteorológica para variáveis radiação solar (Figura 4f) e temperatura do ar (Figura 4g), para as quais a linha de tendência é bastante semelhante à reta $1: 1$, com $\mathrm{R}^{2}$ iguais a 0,9297 e 0,9774 , respectivamente, e d1 superiores a 0,80 . Também fica claro a baixa concordância entre os dados derivados de satélites/modelos e de superfície diários de umidade relativa $(\mathrm{d} 1=0,10)$, que apresenta $\mathrm{R}^{2}$ igual a 0,1574 , distanciando-se da reta 1:1 (Figura 4c). 


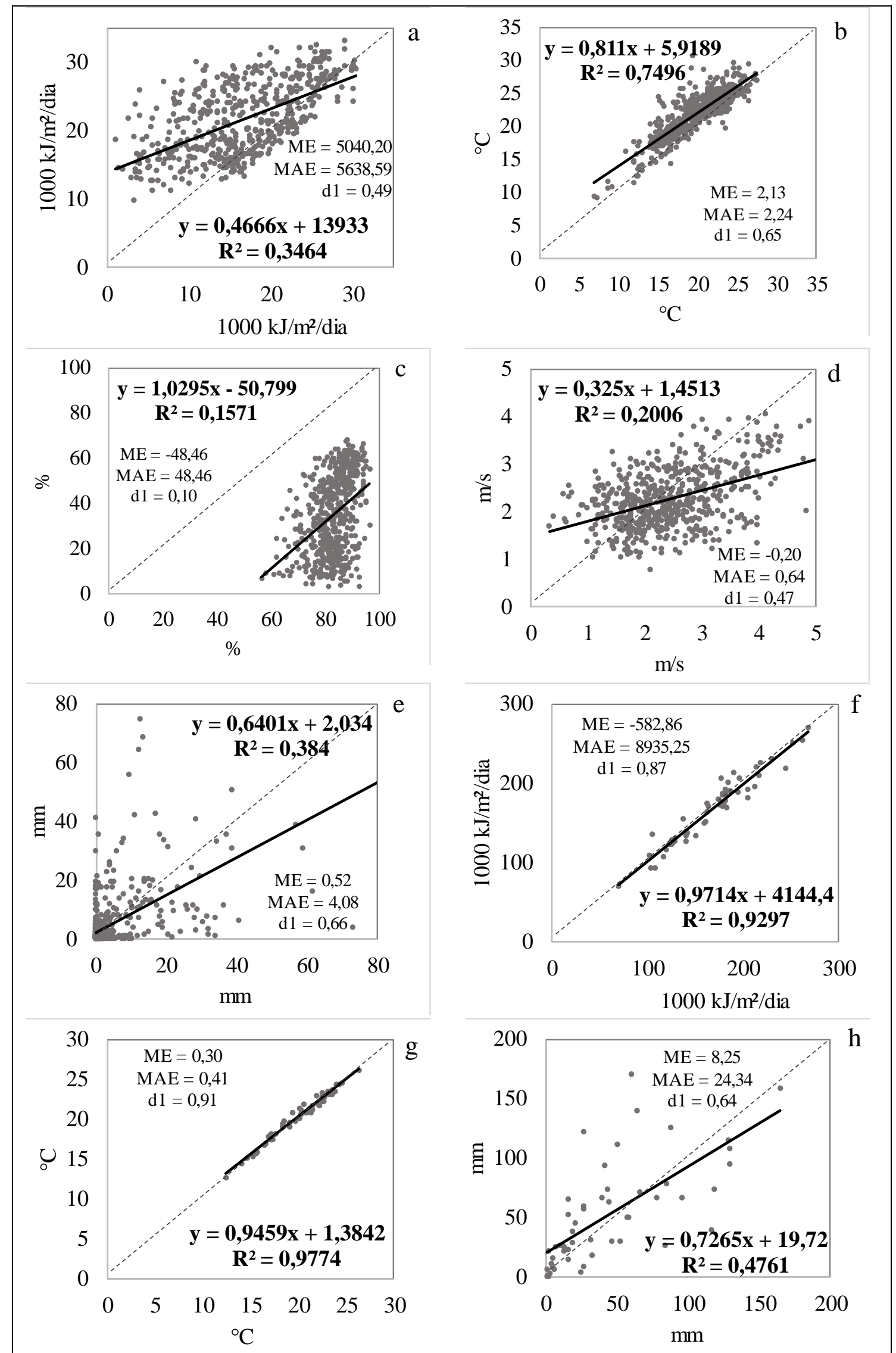

Figura 4. Exemplos dos modelos de RLS para o ponto 10 (São Miguel Arcanjo): dados diários de radiação solar (a), temperatura do ar (b), umidade relativa (c), velocidade do vento (d) e precipitação (e), e dados 
decendiais de radiação solar (f), temperatura do ar (g) e precipitação (h). A linha contínua é a linha de tendência e a linha pontilhada é a reta 1:1.

A Figura 5 ilustra a comparação entre os dados de estação meteorológica e os dados derivados de produtos de satélites ou modelos para o ponto 10 (São Miguel Arcanjo), para as escalas temporais diária e decendial. A análise da Figura 5 confirma os resultados obtidos nos testes estatísticos, discutidos anteriormente: apresentaram concordância satisfatória entre as fontes comparadas os dados diários de radiação solar $(\mathrm{d} 1=0,46$ e 0,49 , respectivamente valor médio e para o ponto 10 ), temperatura do ar $(\mathrm{d} 1=0,63$ e 0,65 , respectivamente valor médio e para o ponto 10), velocidade do vento $(\mathrm{d} 1=0,36$ e 0,47 , respectivamente valor médio e para o ponto 10$)$ e precipitação $(\mathrm{d} 1=0,65$ e 0,66 , respectivamente valor médio e para o ponto 10) e dados decendiais de radiação solar $(\mathrm{d} 1=0,73$ e 0,87 , respectivamente valor médio e para o ponto 10), temperatura do ar $(\mathrm{d} 1=0,82$ e 0,81 , respectivamente valor médio e para o ponto 10$)$ e precipitação $(\mathrm{d} 1=$ 0,66 e 0,64 , respectivamente valor médio e para o ponto 10), enquanto o dado diário de umidade relativa (Figura 5c) apresentou os piores resultados $(\mathrm{d} 1=0,17$ e 0,10 , respectivamente valor médio e para o ponto 10), sendo subestimado em todos os pontos analisados.
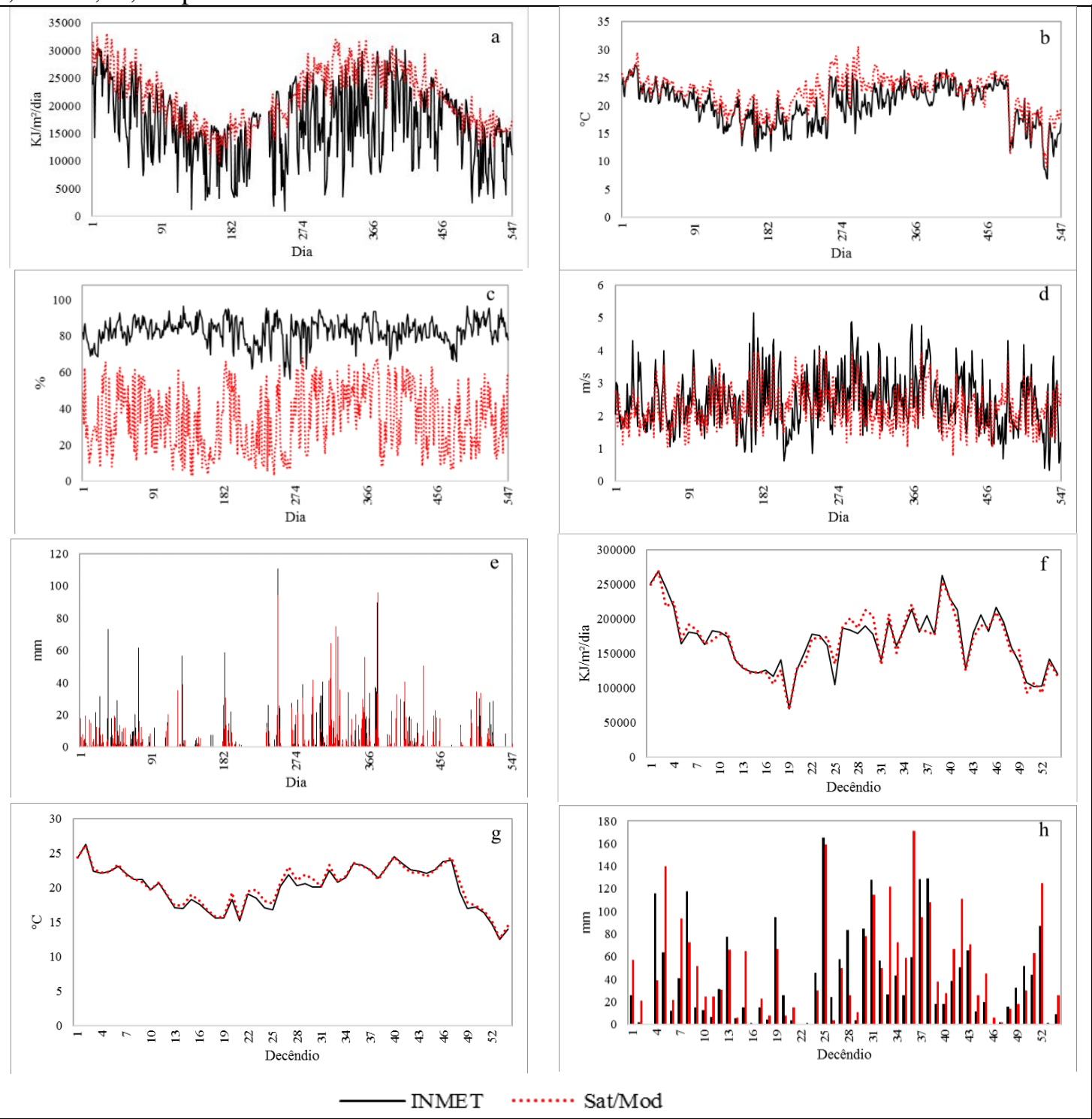

Figura 5. Comparação entre os dados observados em estação meteorológica e os dados derivados de satélites e modelos para o ponto 10 (São Miguel Arcanjo): radiação solar diária (a), temperatura do ar diária (b), umidade relativa diária (c), velocidade do vento diária (d), precipitação diária (e), radiação solar decendial (f), temperatura do ar decendial (g) e precipitação decendial (h) 


\section{Conclusão}

Os resultados obtidos através dos testes estatísticos indicaram que as estimativas fornecidas pelos produtos ou dados de modelos diários de radiação solar (CERES), temperatura do ar (GLDAS), velocidade do vento (GLDAS) e precipitação (PERSIANN), bem como os dados modelados decendiais (ECMWF) de radiação solar, temperatura do ar e precipitação são alternativas adequadas para aplicação em estudos climáticos, já que suprem a falta de informação convencional e, assim, permitem a análise da variabilidade temporal e espacial de elementos meteorológicos, possibilitando um planejamento agrícola mais adequado - o que é primordial para o estado de São Paulo, devido à extensão da área ocupada por atividades agrícolas. A umidade relativa (CM SAF) não apresentou boa exatidão aos dados de estação meteorológica, o que indica que este é um produto que deve ser melhorado para garantir a validade de estudos que o utilizem como parâmetro, ou então que os dados passem por calibração antes de seu uso.

\section{Agradecimentos}

Agradecemos ao INMET pela disponibilização dos dados das estações meteorológicas do estado de São Paulo; ao Conselho Nacional de Desenvolvimento Científico e Tecnológico (CNPq) pela bolsa de estudos (NR); e ao pesquisador Dr. Gabriel Constantino Blain, do Instituto Agronômico de Campinas (IAC).

\section{Referências}

Allen, R. G.; Pereira, L. S.; Raes, D.; Smith, M. 1998, Crop evapotranspiration. Guidelines for computing crop water requirements. FAO Irrigation and Drainage Paper 56, pp300.

Andrade, R. G.; Teixeira, A. H. C.; Leivas, J. F.; Nogueira, S. F.,2016, Analysis of evapotranspiration and biomass in pastures with degradation indicatives in the Upper Tocantins River Basin, in Brazilian Savanna. Revista Ceres 63, 754-760.

Baba, R. K.; Costa, J.; VAZ, M. S. M. G. 2014 Correção de dados agrometeorológicos utilizando métodos estatísticos. Revista Brasileira de Meteorologia (Impresso), 29, 515526.

Blain, G.C.; Rolim, G.de S.; Camargo, M.B.P.; Brunini, O. Relação entre os dados meteorológicos ECMWF preparados pelo JRCMARS-FOOD e dados de superfície para estimativa de produtividade de soja no Estado de São Paulo, Brasil. In: $2^{\circ}$ International Workshop on Crop Monitoring and Forecasting in South America, 2006. Proceedings... Montevideo: South America Scientific Network on Crop Monitoring and Forecasting, 2006, 61-68.

Camparotto, L. B.; Blain, G. C.; Giarolla, A.; Adami, M.; Camargo, M. B. P. 2013 de. Validação de dados termopluviométricos obtidos via sensoriamento remoto para o Estado de São Paulo. Revista Brasileira de Engenharia Agrícola e Ambiental (Online) 17, 665-671.

Davies, J.A. 1967, A note on the relationship between net radiation and solar radiai-o. Quarterly Journal of the Royal Meteorological Society 93, 109-115.

Decker, M.; Brunke, M.A.; Wang, Z.; Sakaguchi, K.; Zeng, X.; Bosilovich, M.G. 2012. Evaluation of the Reanalysis Products from GSFC, NCEP, and ECMWF Using Flux Tower Observations. Journal of Climate, 25, 19161944.

De Gonçalves, L. G. G.; Shuttleworth, W. J.; Nijssen, B.; Burke, E. J.; Marengo, J. A.; Chou, S. C.; Houser, P.; Toll, D. 2006, Evaluation of model-derived and remotely sensed precipitation products for continental South America. Journal of Geophysical Research, $111,16113$.

Depiné, H.; Castro, N. M. R.; Pedrollo, O. C. 2014. Preenchimento de falhas de dados horários de precipitação utilizando redes neurais artificiais. Revista Brasileira de Recursos Hídricos, 51-63.

Dessler, A. E. 2010. A Determination of the Cloud Feedback from Climate Variations over the Past Decade. Science, 330, 1523-1527. DOI: 10.1126.

Eumetsat (European Organisation for the Exploitation of Meteorological Satellites). Satellite Application Facility on Climate Monitoring (CM SAF). Disponível em: $<$ http://www.eumetsat.int/website/home/Satelli tes/GroundSegment/Safs/ClimateMonitoring/i ndex.html>. Acesso em 05 mai. 2017.

Guha-sapir, D.; Hoyois, P.; Below, R. Annual Disaster Statistical Review 2014: The Numbers and Trends. Brussels: CRED. 
Haiden, T.; Janousek, M.; Bidlot, J.; Ferranti, L.; Prates, F.; Vitart, F.; Bauer, P.; Richardson, D.S. Evaluation of ECMWF forecasts, including the 2016 resolution upgrade. ECMWF Technical Memorandum, European Centre for Medium-Range Weather Forecasts

Hsu, K.; Gao, X.; Sorooshian, S.; Gupta, H. V. 1997, Precipitation estimation from remotely sensed information using artificial neural networks. J. Appl. Meteor. Climatol. 36, 1176-1190.

IBGE (Instituto Brasileiro de Geografia e Estatística). Síntese de informações sobre o estado de São Paulo. Censo, 2010. Disponível em:

<http://www.ibge.gov.br/estadosat/perfil.php?s igla=sp >. Acesso em 05 mai. 2017.

Produção Agrícola Municipal 2015: culturas temporárias e permanentes. IBGE. Rio de Janeiro, RJ, v. 42, 2015. Disponível em: <http://www.ibge.gov.br/home/estatistica/econ omia/pam/2015/default.shtm>. Acesso em 05 mai. 2017.

INMET (Instituto Nacional de Meteorologia). Normais climatológicas do Brasil 1961-1990. Organizadores: Andrea Malheiros Ramos, Luiz André Rodrigues dos Santos, Lauro Tadeu Guimarães Fortes. Brasília, DF: INMET, 465.

INMETRO (Instituto Nacional de Metrologia, Qualidade e Tecnologia). Vocabulário Internacional de Metrologia - VIM 2012: Conceitos Fundamentais e Gerais e Termos Associados. Rio de Janeiro, $1^{\mathrm{a}}$ edição lusobrasileir.

Johann, J. A.; Rocha, J.; Rolim, G. de S.; Lamparelli, R. A. C.; DUFT, D. G. Comparação entre dados de temperatura média de estações meteorológicas com no Estado do Paraná por meio de índices de vegetação do sensor MODIS. In: XV Simpósio Brasileiro de Sensoriamento Remoto - SBSR, 2011, Curitiba - PR. Anais... XV Simpósio Brasileiro de Sensoriamento Remoto - SBSR 2011. São José dos Campos - SP: Instituto Nacional de Pesquisas Espaciais - INPE. v. único. 58175824.

JRC (Joint Research Centre). ECMWF Agrometeorological Data. Disponível em: $<$ http://spirits.jrc.ec.europa.eu/>. Acesso em 03 mai. 2017.

Liska, G.R.; Bortolini, J.; Sáfadi, T.; Beijo, L.A. 2013, Estimativas de velocidade máxima de vento em Piracicaba-SP via Séries Temporais e Teoria de Valores Extremos. Revista Brasileira de Biometria, São Paulo 31, 295-309.

Martinelli, M. 2009, Relevo do Estado de São Paulo. Confins (Paris) 7, 1-26.

Moazami, S.; Golian, M. S.; Kavianpour, R., Hong. Y. 2013, Comparison of PERSIANN and V7 TRMM Multi-satellite Precipitation Analysis (TMPA) products with rain gauge data over Iran. International Journal of Remote Sensing, 34, 8156-8171.

Rodell, M.; Houser, P. R.; Jambor, U.; Gottschalck, J.; Mitchell, K.; Meng C-J.; Arsenault, K.; Cosgrove, B.; Radakovich, J.; Bosilovich, M.; Entin, J. K.; Walker, J. P.; Lohmann, D.; TOLL, D. 2004. The Global Land Data Assimilation System. Bull. Am. Meteorol. Soc. 85, 381-394.

Schulz, J.; Albert, P.; BEHR, H. D. 2009. Operational climate monitoring from space: The EUMETSAT Satellite Application Facility on Climate Monitoring (CMSAF), J. Atmos. Chem. Phys., 9, 1687-1709.

Rolim, G.; Camargo, M.; Lania, D.; Moraes, J. 2007, Classificação climática de Köppen e de Thornthwaite e sua aplicabilidade na determinação de zonas agroclimáticas para o estado de são Paulo. Bragantia. Instituto Agronômico de Campinas, 66, 711-720,

Shapiro, S. S.; Wilk, M. B. 1965. An Analysis of Variance Test for Normality (Complete Samples). Biometrika Trust, London, 52, 591609.

Spearman, C. 2904, The proof and measurement of association between two things. American Journal of Psychology, 15, 72-101.

Szewczyk, Z. P.; Smith, G. L.; Priestley, K. J. 2005. Validation of Clouds and Earth Radiant Energy System instruments aboard the Terra and Aqua satellites. Journal of Geophysical Research: Atmospheres, 110.

Tiba, C.; Fraidenraich, N.; Moszkowicz, M.; CavalcantI, E. S. C.; Lyra, F. J. M.; Nogueira, A. M. B.; GROSSI, H. G. 2001, Atlas Solarimétrico do Brasil. Recife, Pernambuco: Editora Universitária - UFPE, pp 111.

Wielicki, B. A.; Barkstrom, B. R.; Harrison, E. F.; Lee III, R. B.; Smith, G. L.; Cooper, J. E. 1996. Clouds and the Earth's Radiant Energy System (CERES): An Earth observing system 
experiment. Bull. Am. Meteorol. Soc. 77, 853868.

Wielicki, B.A.; Wong, T.; Allan, R.P.; Slingo, A.; Kiehl, J.T.; Soden, B.J.; Gordon, C.T.; Miller, A.J.; Yang, S.K.; Randall, D.A.; Robertson, F.; Susskind, J.; Jacobowitz, H. 2002. Evidence for Large Decadal Variability in the Tropical Mean Radiative Energy Budget. Science, 295, 841844, DOI: 10.1126.

Willmott, C. J. 1981. On the validation of models. Physical Geography, 2, 184-194.
Willmott, C. J.; ackleson, S. G.; Davis, J. J.; Feddema, K. M.; Klink, D. R. 1985. Statistics for the evaluation and comparison of models. Journal of Geophysical Research, 90, 89959005.

Xavier, A. C.; King, C. W.; Scanlon, B. R. 2016. Daily gridded meteorological variables in Brazil (1980-2013). International Journal of Climatology, 36, 2644-2659. 